\title{
Validation of specificity in doping control: problems and prospects
}

\author{
Nicolaas (Klaas) M. Faber
}

Received: 16 May 2009/Published online: 20 June 2009

(C) Springer-Verlag 2009

Sir,

The World Anti-Doping Agency (WADA) has been created in 1999 to improve and harmonize the global fight against doping [1]. To set the standards for laboratory anti-doping procedures around the world, WADA has developed the International Standard for Laboratories (ISL) [2]. According to the ISL, confirmation methods for non-threshold substances, which comprise the great majority of prohibited substances, must be validated. Specificity is one of the key analytical figures of merit for determining if such a method is fit for intended use. Its proper validation requires (Section 5.4.4.2.1 in [2]) that "The ability of the assay to detect only the substance of interest shall be determined and documented." Experienced analysts will recognize that the word 'only' is of paramount importance here. The purpose of this Letter is to draw attention to the fact that, surprisingly, these methods often only comply with the requirement from which the word 'only' is removed.

A case in point is the recently improved test for metandienone [3]. The authors seem to assume that specificity comes for free with the mass spectrometric identification and characterization of the substance of interest. However, logic dictates that specificity can only be ensured by also considering compounds with closely resembling mass spectra. This has been stressed by de Zeeuw [4] in an article aptly entitled "Substance identification: the weak link in analytical toxicology". Specifically, de Zeeuw reports 19 substances whose mass spectra closely resemble the spectrum of methamphetamine. One might argue that these substances will not be mistaken for methamphetamine, since a chromatographic separation step precedes mass

N. M. Faber $(\bowtie)$

Chemometry Consultancy, Goudenregenstraat 6,

6573 XN Beek-Ubbergen, The Netherlands

e-mail: nmf@chemometry.com spectrometric detection. However, from a scientific point of view, one cannot accept a rather vague assumption to stand proxy for a rigorous validation. Also from a practical point of view, a method being fit for purpose cannot be taken for granted. It follows that studies like the one reported in [3] can only lead to unsubstantiated claims with respect to specificity. This is a serious problem in practice because any lack of specificity remains unnoticed, unless specifically addressed. The proficiency testing (PT) program detailed in [1], for example, will not function as a safeguard since, quite naively, PT samples are not (systematically) designed to contain potential interferents!

The correct state-of-the-art scientific approach is to search in an extensive library for candidate interferents, and subsequently scrutinize these candidates for co-elution. This search solves certain fundamental problems and also offers valuable benefits. For example, current selection of mass spectrometric signals for detection is commonly based on subjective 'visual inspection', see [3] for an illustration. This ad hoc approach could be replaced by a suitable objective (computerized) optimization strategy, which is the standard in fields like statistics and chemometrics. Method improvement and optimization easily come within reach.

\section{References}

1. Ivanova V, Boghosian T, Rabin O (2007) Accred Qual Assur 12:491-493. doi:10.1007/s00769-007-0283-4

2. The International Standard for Laboratories (version 5.0, January 2008). WADA, Montreal, Canada. http://www.wada-ama.org/ rtecontent/document/lab_08_V_05.pdf

3. Schänzer W, Geyer H, Fußhöller G, Halatcheva N, Kohler M, Parr M-K, Guddat S, Thomas A, Thevis M (2006) Rapid Commun Mass Spectrom 20:2252-2258. doi:10.1002/rcm.2587

4. de Zeeuw RA (2004) J Chromatogr B Analyt Technol Biomed Life Sci 811:3-12 